\title{
The odd case when cognitive biases are more prevalent in researchers than in samples
}

Jose D. Perezgonzalez

Massey University, New Zealand

\section{Abstract}

Walmsley and Gilbey (2016) reported on the impact of cognitive biases on pilots' decisionmaking, concluding that there was strong evidence that cognitive bias impacted decision making thus putting pilots' lives in danger. However, their methodology was not free of the same biases they set to research and, more importantly, they relied far too much on statistical significance as the only standard for result interpretation. Consequently, while the results obtained may have been technically correct, their divorce from the underlying methodological context made them factually wrong. Therefore, the conclusions achieved also misrepresented the true impact of cognitive biases on pilots' decision-making.

Keywords: cognitive biases, anchoring and adjustment effect, confirmation bias, outcome bias, significance testing, visual flight rules, instrument meteorological conditions 


\section{The odd case when cognitive biases are more prevalent in researchers than in samples}

Walmsley and Gilbey (2016; based on Walmsley, 2016 ${ }^{1}$ ) reported on the impact of cognitive biases on pilots' decision-making, concluding that their findings "provide strong evidence" of such impact and that the effects were "likely to place pilots in greater danger" (p. 542). The research was picked up by news outlets (e.g., Staufenberg , 2016) and social media (e.g., Fradera, 2016) with a spin-off into the irrationality of pilots and flight safety. Despite all said, however, the original research seems more informative about the cognitive biases of the researchers than of their sample, as subsequently discussed.

In Study 1, on the anchoring and adjustment effect, the authors declared that pilots exposed to the high anchor "reported a higher assessment of cloud height...( $M=2468 \ldots)$, visibility...( $M$ $=15.50 \mathrm{~km} . .$.$) , [and] likelihood of deciding to assess conditions as safe enough to continue$ the flight...( $(M=6.62 \ldots)$ compared to when they had been exposed to the low anchor”, thus "failing to adjust their perceptions in the face of evidence to the contrary...[and] more likely to feel that it was safe to continue the flight”, particularly experienced pilots (p. 535-536). And yet, all scenarios were designed with a cloud base at 2500 feet and $16 \mathrm{~km}$ of visibility, adequate for VFR (visual flight rules) flight. Therefore, participants exposed to the high anchor perceived the experimental conditions accurately and their higher likelihood to continue flying into objectively safe VFR conditions was not irrational. Only the participants exposed to the low anchor showed bias, heavily discounting the observable evidence and,

\footnotetext{
${ }^{1}$ Authorship conventions dictate that authors are solely responsible for the contents they publish, irrespective of sources or influences. This said, I was co-supervisor of Walmsley's thesis and, therefore, may have had some influence-or lack of, thereof-on Walmsley's work. As far as I may bear some responsibility as a 'ghost' author of Walmsley's thesis, the criticisms laid here may equally apply to me retrospectively, and shall serve as a partial correction of the research literature cited (also BetterScience, 2016, Perezgonzalez, 2016, 2017).
} 
worryingly, preferring to continue their flight $(M=6.17$ on a scale running from ' 1 ' $=$ strongly disagree to ' 9 ' = strongly agree) than to divert.

In Study 2, on the confirmation bias, the authors concluded that there was "no evidence that participants valued disconfirmatory evidence over confirmatory [one]” (p. 539). However, the simple tests of significance they used are not appropriate for ascertaining null hypotheses (Fisher, 1954), making such conclusion unwarranted. They need Neyman-Pearson’s approach (1933) or Bayes factors (Jeffreys, 1961) for such purpose. More simply, though, had they used confidence intervals (CIs, Neyman, 1935; Cumming, 2012; Perezgonzalez, 2015a) they would have observed that some evidence seems apparent but that the tests were not sensitive enough to capture it as a statistically significant result (Figure 1).

FIGURE 1 | Confidence intervals for measures of confirmation bias in pilots

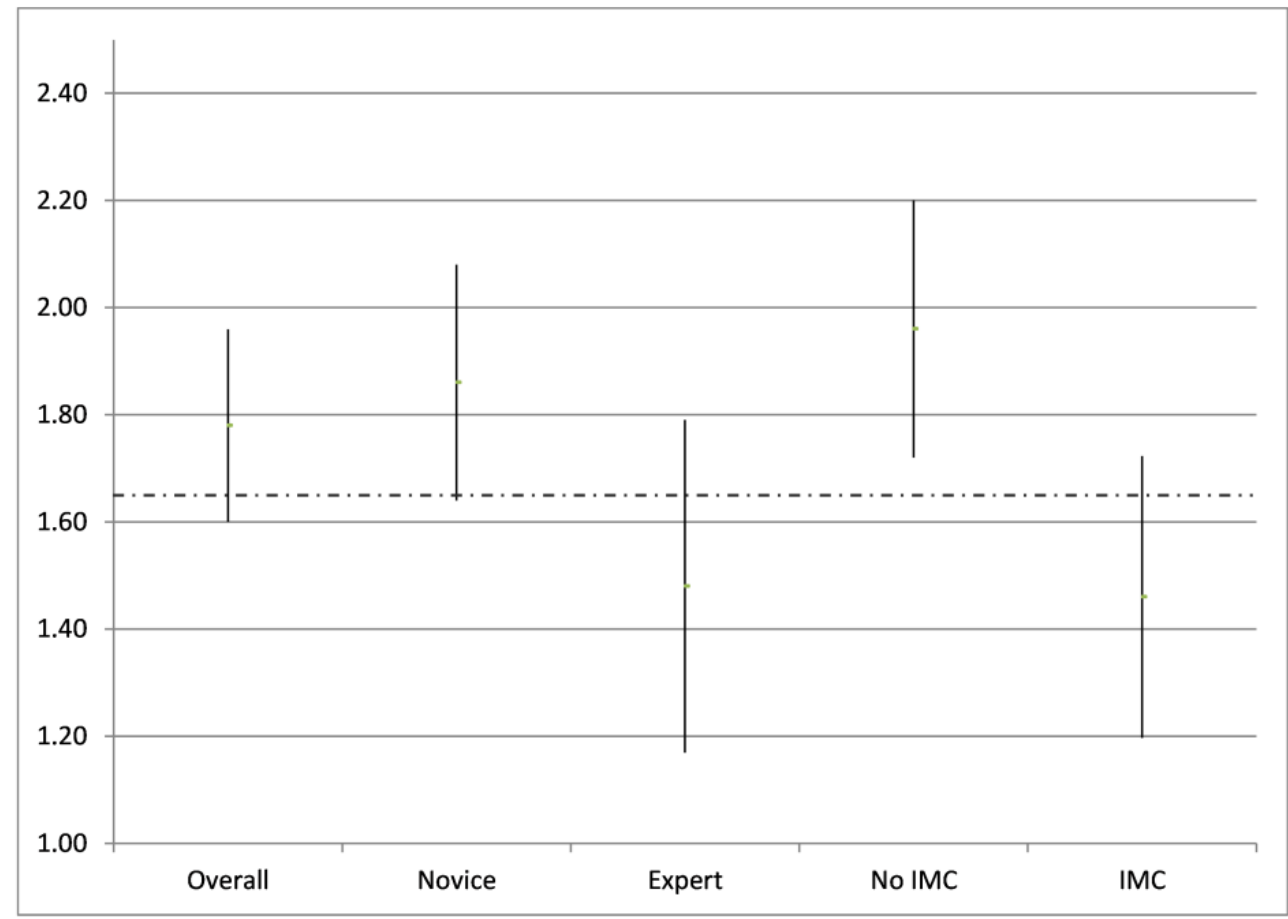

Notes: Overall sample. Novice versus expert pilots. Pilots without versus with experience entering instrument meteorological conditions (IMC). The horizontal line at point 1.66 represents the number of disconfirming choices expected by chance. 
Furthermore, Study 2’s methods are suspect. The disconfirmatory items were largely ambiguous (phrased as 'almost certainly', 'may') for three of the five scenarios while the confirmatory items were certain (phrased as 'can see', 'safely flew'), and adjudication to a confirmation bias was self-serving, as "inappropriate use of the positive testing strategy may put the pilot at risk of VFR flight into IMC [instrument meteorological conditions] and was interpreted as evidence of confirmation bias” (Walsmley, 2016, p. 92). The latter case was compounded by scenarios urging prompt landing either because of developing emergencies (e.g., engine making strange noises) or because of their "it is important” phraseology (e.g., very important meeting but no other aerodromes in the immediate area), with heavy costs in time and opportunity if diverting. Such design may partly account for pilots discounting the ambiguous disconfirming items, while not fencing off alternative rationalizations which expert pilots and those with experience entering inadvertently into IMC may have entertained (such as their confidence in having navigated IMC successfully, or deciding to wait for the situation to become less ambiguous before diverting, or planning an emergency landing as last resort).

Study 3, on outcome bias-which, ironically, the authors showed when designing scenarios "favourable for conducting a VFR flight" yet "based on actual weather reports at the time of real VFR flight into IMC accidents” (Walmsley, 2016, p. 111-113)—was based on group comparisons and found that participants exposed to negative outcomes assessed the situations as being worse than participants exposed to positive outcomes, thus concluding that the latter "were more likely to conduct the same flight themselves...[which] might increase the risks faced... with no guarantee that the outcome will be good” (p. 541).

However, the authors also failed to account for the methodology used: When compared against the standard scale (running from ' 1 ', a favourable assessment, to '9', an unfavourable assessment), the effect of negative outcomes is apparent both for vicarious judgement $(M=$ 
6.02) and vicarious risk assessment $(M=5.96)$, yet positive outcomes show no effect either way ( $M=5.01, M=5.03$, respectively), meaning a neutral assessment or indecision (Figure 2). Thus, while negative outcomes made participants more conservative regarding their vicarious assessments, a positive outcome did not lead to liberal assessments, as implied. More importantly, all groups reported their decision to fly to be safer than that of others (by 0.66 points for the negative outcome participants, by 0.85 for the positive outcome participants, and by 0.57 points for the control group). This illusion of control seems understandable for the positive outcome pilots, as they just showed a neutral assessment of vicarious experience. What is at odds is that pilots with the hindsight of negative vicarious information still showed a similar degree of illusion of control, remaining uncertain regarding to whether they will fly in similar conditions or not (one would expect them to score higher in aversion). The simple use of CIs would also have helped the authors locate the results in the overall context of their own methodology, leading to different conclusions.

Why did the authors go astray in their conclusions? I do not believe the authors were tempting Huff (1954) or Goldacre (2008), nor simply scaremongering. Instead, their methodology was not completely free of biases (confirmation and outcome biases) and, more importantly, they relied far too mindlessly on statistical significance as the only standard for result interpretation, thus becoming blind to their own methods and observed data, a cognitive bias of anchoring on a misunderstood approach to testing (Gigerenzer, 2004; Perezgonzalez, 2015b). Consequently, while the tests results may have been technically correct, their divorce from the underlying methodological context made them factually wrong, as so were the conclusions attained. 


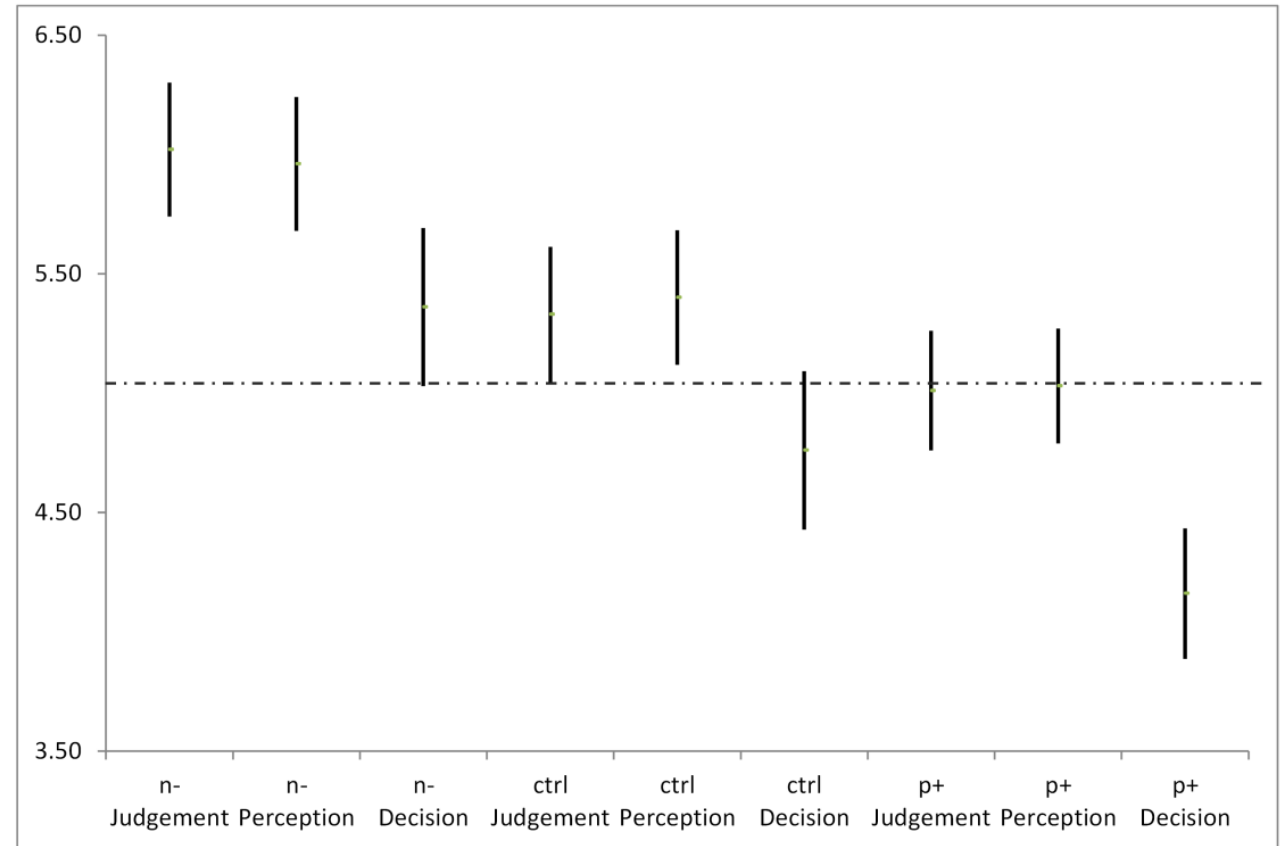

Notes: n-, negative outcomes condition; ctrl, control group; $\mathrm{p}^{+}$, positive outcomes condition. Judgement, vicarious judgement of risk; Perception, vicarious assessment of risk; Decision, own assessment of willingness to flight under similar conditions. Horizontal line represents the neutral point on a nine-anchor scale. 


\section{References}

BetterScience. (2016, December 22). Re: Pilots 'very likely' to misjudge flying conditions due to irrational decisions [Online comment to news article]. Retrieved from http://www.independent.co.uk/news/science/pilots-very-likely-to-misjudge-flyingconditions-due-to-irrational-decisions-psychology-study-a7033481.html. Pre-print available as doi:10.6084/m9.figshare.4490789.

Cumming, G. (2012). Understanding the new statistics. Effect sizes, confidence intervals, and meta-analysis. New York, NY: Routledge.

Fisher, R. A. (1954). Statistical methods for research workers (12th edn). Edinburgh, UK: Oliver and Boyd.

Fradera, A. (2016, May 16). Sorry to say, but your pilot's decisions are likely just as irrational as yours and mine [Web log post]. Retrieved from http://digest.bps.org.uk/2016/05/sorry-to-say-but-your-pilots-decisions.html

Gigerenzer, G. (2004). Mindless statistics. The Journal of Socio-Economics 33, 587-606. doi:10.1016/j.socec.2004.09.033

Goldacre, B. (2008). Bad science. London, UK: Fourth Estate.

Huff, D. (1954) How to lie with statistics. New York, NY: W.W. Norton.

Jeffreys, H. (1961). Theory of probability (3rd edn). Oxford, UK: Oxford University Press. Neyman, J. (1935). On the problem of confidence intervals. The Annals of Mathematical Statistics 6(3), 111-116. doi:10.1214/aoms/1177732585

Neyman, J., \& Pearson, E. S. (1933). On the problem of the most efficient tests of statistical hypotheses. Philosophical Transactions of the Royal Society of London. Series A 231, 289-337. doi:10.1098/rsta.1933.0009

Perezgonzalez, J. D. (2015a). Confidence intervals and tests are two sides of the same research question. Frontiers in Psychology 6:34. doi:10.3389/fpsyg.2015.00034 
Perezgonzalez, J. D. (2015b). Fisher, Neyman-Pearson or NHST? A tutorial for teaching data testing. Frontiers in Psychology 6:223. doi:10.3389/fpsyg.2015.00223

Perezgonzalez, J. D. (2016, December 16). Re: Sorry to say, but your pilot's decisions are likely just as irrational as yours and mine [Web log comment]. Originally available at https://digest.bps.org.uk/2016/05/16/sorry-to-say-but-your-pilots-decisions-are-likelyjust-as-irrational-as-yours-and-mine/comment-page-1/\#comment-10868 (All comments removed by BPS Research Digest, on 2017, January 24). Pre-print retrievable as doi:10.6084/m9.figshare.4460078

Perezgonzalez, J. D. (2017, February). The luxury of living in the Ivory Tower. New Zealand Aviation News, p. 20.

Walmsley, S. (2016). Flights into deteriorating weather conditions: Investigating cognitive biases in weather-related decision making (Doctoral thesis, Massey University, Manawatu, New Zealand). Retrieved from http://hdl.handle.net/10179/8275

Walmsley, S., \& Gilbey, A. (2016). Cognitive biases in visual pilots' weather-related decision making. Applied Cognitive Psychology 30(4), 532-543. doi:10.1002/acp.3225

Staufenberg, J. (2016, May 18). Pilots 'very likely' to misjudge flying conditions due to irrational decisions. The Independent. Retrieved from http://www.independent.co.uk/news/science/pilots-very-likely-to-misjudge-flyingconditions-due-to-irrational-decisions-psychology-study-a7033481.html 